\title{
Limit analysis of cracked structure by combination of extended finite element method with linear matching method
}

\author{
Jun-Hyok $\mathrm{Ri}^{\mathrm{a}}$ and Hyon-Sik Hong ${ }^{\mathrm{a}^{*}}$
}

${ }^{a}$ Institute of Mechanics, State Academy of Sciences, Pyongyang, DPR of Korea

\begin{tabular}{l}
\hline A R T I C L EI N F O \\
\hline Article history: \\
Received 26 October, 2017 \\
Accepted 9 January 2018 \\
Available online \\
9 January 2018 \\
\hline Keywords: \\
Limit analysis \\
Linear matching method (LMM) \\
Extended finite element method \\
(XFEM) \\
Linear elastic fracture mechanics \\
(LEFM) \\
Finite element analysis (FEA)
\end{tabular}

\section{Introduction}

The mathematical modeling of limit analysis reduces to the nonlinear optimization problem with infinite constraints, in which finite element method is used for replacing infinite constraints by finite constraints (Casciaro \& Garcea, 2002). Numerical methods for solving the optimization problem of limit analysis can be classified into direct method and indirect method. The sequential programming and the second cone programming is one of typical mathematical programming used for solving the

* Corresponding author.

E-mail addresses: honghs501@yahoo.com (H.-S. Hong) 
optimization problem, respectively. These methods are usually combined with the base reduction method in order to reduce the computational cost. By using only the finite element code, it could be impossible to implement the limit analysis due to the necessity of combination of FEA with mathematical programming in spite that some studies did limit analysis by using the general finite element package PERMAS (Staat \& Heitzer, 2003). LMM is one of the indirect methods, which does not solve the optimization problem of limit analysis directly as demonstrated (Chen \& Ponter, 2001). LMM has been widely used for engineering applications including shakedown and limit analysis of the cracked bodies subjected to cyclic load and temperature (Habibullah \& Ponter, 2005; Chen et al., 2011), integrity assessment of 3D tube plate (Chen \& Ponter, $2005 \mathrm{a}, \mathrm{b}$ ), shakedown and limit analyses applied to rolling contact problem (Chen \& Ponter, 2005c), evaluation of fatigue-creep and plastic collapse of notched bars (Ponter et al., 2004), evaluation of plastic and creep behaviors for bodies subjected to cyclic thermal and mechanical loading (Chen \& Ponter, 2001), structural integrity assessment of super heater outlet penetration tube plate (Chen \& Ponter, 2009), shakedown and limit analysis of particulate metal matrix composite (Barrera et al., 2011), limit analysis of orthotropic laminates (Fuschi et al., 2009) and creep-fatigue strength of welded joints (Gorash \& Chen, 2013).

LMM has an advantage of being of extracting the converged upper series of linear elastic solutions iteratively, leading to simple implementation by using UMAT of ABAQUS (Chen \& Ponter, 2009). Even though LMM is one of the effective numerical methods for the shakedown and limit analysis, its numerical accuracy depends on conventional finite element method since the mechanical quantities like stress and strain should be evaluated by FEM itself. Thus, LMM has the same limitations as in the conventional FEM for discontinuous problems such as crack problem. In order to overcome such a difficulty, some authors raised the mesh adaptive strategies of upper and lower bounds in limit analysis, where assess the convergence based on results of simultaneous upper and lower analysis, leading to the re-meshing for iterative computation (Ciria et al., 2008; Munoz et al., 2009). Some authors suggested the lower-bound limit analysis by using meshless element-free Galerkin (EFG) and non-linear programming that is unlike standard FEM (Chen et al., 2008; Le et al., 2009, 2010). Nevertheless, it is widely accepted that XFEM is more effective for crack problem as compared with EFG.

XFEM enriches the approximation space of standard finite element method by adding specific functions reflecting characteristics of given problems into the standard finite element approximation based on the partition unity (PU), keeping well-known standard framework of conventional FEM (Fries \& Belytschko, 2010; Belytschko \& Black, 1999; Moës et al., 1999). In such a way, the property of specific problem can be reflected on finite element solution. XFEM has been applied successfully for 2D and 3D crack analysis (Sukumar \& Prévost, 2003; Huang et al., 2003; Moës et al., 2002; Gravouil et al., 2002).

Authors think that the numerical accuracy of limit analysis for cracked structures may be improved significantly by combining XFEM with LMM due to advantages of XFEM and LMM for accessing crack tip singularity. Thus, attention will be paid to the combination of XFEM with LMM for the limit analysis of cracked bodies and the validation of its numerical accuracy for plane problem in this paper. The proposed method is very straightforward and XFEM and LMM will be implemented by using ANSYS subroutine UserElem for definition of user-element as well as ANSYS subroutine UserMat for definition of user-material. Numerical validation is done for two types of typical fracture specimens. Numerical examples show that the limit analysis by combining XFEM with LMM gives more accurate result compared with the one by combining of conventional FEM with LMM. Furthermore, we demonstrated that the choice of enrichment region plays an important role in the improvement of numerical accuracy in the proposed method of this paper.

The contents of this paper are as follows. Section 2 gives a main idea of the LMM and its iterative algorithm. Section 3 describes a brief description of how XFEM is formulated for modeling cracks. Section 4 explains the implementation of limit analysis by the combination of LMM with XFEM. 
Numerical results for some crack specimens are presented in section 5. A few concluding remarks are mentioned in section 6 .

\section{Linear matching method for limit analysis}

\subsection{Lower and upper bound theorem of limit analysis}

Let us consider the limit analysis of perfectly-plastic material which follows von Mises yielding rule. It is assumed that a body occupies volume $V$ with surface $S$ where a traction is given as zero or $P \cdot p_{i}(\mathbf{x})$ on $S_{T}$ and displacement $\bar{u}_{i}=0$ is specified on $S_{u}\left(S=S_{T}+S_{u}\right)$. Here, $P$ is a scalar parameter defining relative magnitude of applied load as compared with a reference load $p_{i}$. The lower and upper bound theorem of limit load can be postulated as follows, respectively (Staat \& Heitzer, 2003).

\section{Lower bound theorem:}

If, for the external load $P=P_{L B}$, there exists a statically possible stress field $\sigma_{i j}^{*}$ such that

$$
f\left(\sigma_{i j}^{*}\right) \leq \sigma_{y}
$$

at every point within $V$, then $P_{L} \geq P_{L B}$. Here, $f$ is a von Mises yield function and $\sigma_{y}$ is an uniaxial yield stress. Thus, one can know that a maximum value of $P_{L B}$ becomes lower bound of limit load.

\section{Upper bound theorem:}

If, for the external load $P=P_{U B}$, there exists a kinetically possible displacement rate field $\dot{u}_{i}^{*}$ and its corresponding strain rate field $\dot{\varepsilon}_{i j}^{*}$ such that

$$
P_{U B} \int_{S_{T}} p_{i} \dot{u}_{i}^{*}=\int_{V} \sigma_{i j}^{p^{*}} \dot{\varepsilon}_{i j}^{*} d V
$$

where $\sigma_{i j}^{p^{*}}$ is a stress point at yield associated with $\dot{\varepsilon}_{i j}^{*}$, then satisfies $P_{L} \leq P_{U B}$. Hence, one can know that a minimum value of $P_{U B}$ becomes upper bound of limit load.

According to above theorems, lower and upper bound of the limit load could be evaluated by solving the optimization problem. The direct method employs the optimization approach based on the admissible space as the statically possible stress filed or the kinetically possible strain field, while the indirect method obtains the statically possible stress filed or the kinetically possible strain field by using FEA with a certain special material property, leading to the improvement of solution of limit load. LMM takes the kinetically possible strain field as the FEA solution obtained adjusting spatially varying material properties.

\subsection{LMM algorithm for limit analysis}

As mentioned above, LMM is based on the linear elastic FEA with spatially varying material properties. The Young's modulus is changed spatially such that stress field corresponding to a certain kinetically possible strain field is placed on the yielding surface at every point of material. The Poisson ratio is taken as 0.4999999 since material is considered to have plastic incompressibility. LMM algorithm could be formulated as follows (Chen \& Ponter, 2001).

- Initialization: Set $P_{U B}^{0}=1, E^{1}(\mathbf{x})=E$.

- $k$ th iteration: 
The linear elastic analysis with the Young's modulus of $E^{k}(\mathbf{x})$ is performed under a load $P_{U B}^{k-1} \cdot p$ and $\sigma_{i j}^{k}, \varepsilon_{i j}^{k}$ and $u_{i}^{k}$ is obtained, respectively. Lower and upper bound of the limit load at $k$ th iteration is evaluated as

$$
\begin{aligned}
& P_{L B}^{k}=P_{U B}^{k-1} \frac{\sigma_{y}}{\sigma_{e q}\left(\sigma_{i j}^{k}\right)}, \\
& P_{U B}^{k}=P_{U B}^{k-1} \frac{\int_{V} \sigma_{y} \varepsilon_{e q}\left(\varepsilon_{i j}^{k}\right)}{\int_{S_{T}} P_{U B}^{k-1} p_{i} u_{i}^{k}},
\end{aligned}
$$

where, $\sigma_{e q}$ and $\varepsilon_{e q}$ denote equivalent stress and equivalent strain, respectively.

$E^{k+1}$ at $k+1$ th iteration is updated as follows,

$$
E^{k+1}=\frac{\sigma_{y}}{\varepsilon_{e q}\left(\varepsilon_{i j}^{k}\right)}
$$

Eq. (5) gives $E^{k+1}$ at $k+1$ th iteration such that stress field corresponding to strain field $\varepsilon_{i j}^{k}$ obtained at $k$ th iteration lies on the yielding surface. Nevertheless, for high gradient of stress, Young's modulus evaluated by Eq. (5) may not give stable solution, sometimes. In order to overcome this numerical difficulty without any affection on LMM solution, we do the normalization using initial Young's modulus $E_{\text {ref }}$ of material.

We denote a minimum value of Young's modulus $E^{k}(\mathbf{x})$ on whole region obtained at $k-1$ th iteration by $E_{\min }^{k}=\min _{\mathbf{x}} E^{k}(\mathbf{x})$. Then, after performing $k$ th iteration, $E^{k+1}$ at $k+1$ th iteration will be evaluated by

$$
E^{k+1}=\frac{E_{r e f}}{E_{\min }^{k}} \frac{\sigma_{y}}{\varepsilon_{e q}\left(\varepsilon_{i j}^{k}\right)}
$$

instead of using Eq. (5). Even though Eq. (6) shows a theoretical equivalence with Eq. (5), our computational experiences ensure that Eq. (6) can improve the numerical stability much more as compared with Eq. (5).

\section{XFEM for linear elastic crack}

Two types of functions, namely, Heaviside function considering displacement discontinuity on crack faces and functions taking account for characteristics of crack tip displacement fields are enriched into standard finite element approximation for modeling cracks in XFEM approach. By using the partition of unity method (PUM), displacement fields are interpolated as

$$
\mathbf{u}^{h}(x)=\sum_{i \in I} N_{i}(\mathbf{x}) \mathbf{u}_{0 i}+\sum_{j \in J} N_{j}(\mathbf{x}) H(\mathbf{x}) \mathbf{a}_{j}+\sum_{k \in M} N_{k}(\mathbf{x})\left(\sum_{\alpha} F^{\alpha}(\mathbf{x}) \mathbf{b}_{k}^{\alpha}\right),
$$

where $I$ is a set containing all nodes in finite element model, $J$ a subset involving nodes enriched by Heaviside function and $M$ a subset having nodes enriched by crack tip enrichment functions (Belytschko \& Black, 1999; Moës et al., 1999). A component $N_{i}$ is the shape function of standard finite element method concerning with a node $i$ and $\mathbf{u}_{0_{i}}$ is a corresponding degree of freedom. Factors $\mathbf{a}_{j}$ and $\mathbf{b}_{k}^{\alpha}$ is a degree of freedom related to Heaviside function and crack tip enrichment functions, 
respectively. Heaviside function $H(x)$ is defined as

$$
H(\mathbf{x})= \begin{cases}-1 & \phi(\mathbf{x})<0 \\ +1 & \phi(\mathbf{x}) \geq 0\end{cases}
$$

where $\phi(x)$ denotes the signed distance function from a crack (usually determined by means of the level set method, LSM). Crack tip enrichment function $F^{\alpha}(\mathbf{x})$ is usually chosen as

$$
\left\{F^{\alpha}(\mathbf{x})\right\}_{\alpha=1}^{4}=\left\{\sqrt{r} \cos \left(\frac{\theta}{2}\right), \sqrt{r} \sin \left(\frac{\theta}{2}\right), \sqrt{r} \sin \left(\frac{\theta}{2}\right) \sin (\theta), \sqrt{r} \cos \left(\frac{\theta}{2}\right) \sin (\theta)\right\}
$$

where $r$ and $\theta$ are local crack tip polar coordinates, taking account for the first term concerning with the SIF in crack tip asymptotic displacement fields (Le et al., 2010; Fries \& Belytschko, 2010).

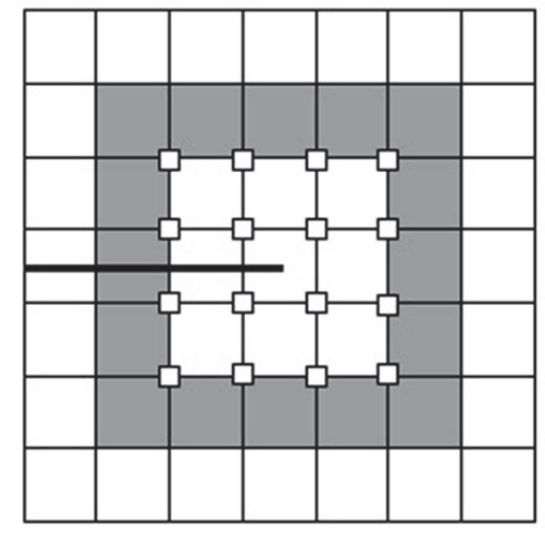

(a)

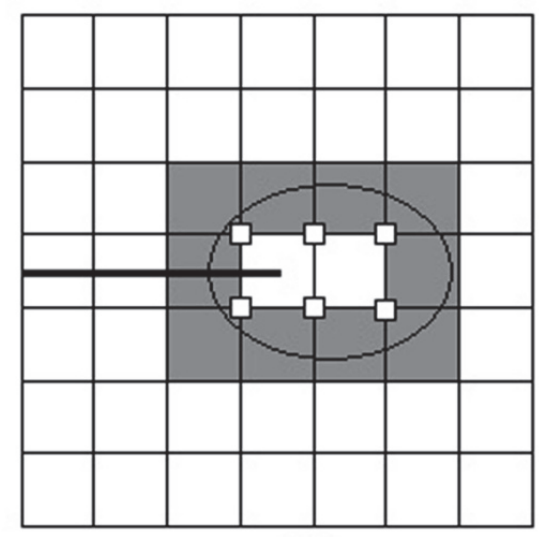

(b)

Fig. 1. (a) Topological enrichment of two layers. (b) Geometrical enrichment

Enriched nodes near a crack tip could be chosen by various ways. First, only elements containing a crack tip are completely enriched with singular functions (Le et al., 2010). With this technique, one or more layers of neighboring elements are added to the enriched region, so that its size is increased. The size of the enriched region is proportional to the size of element. This type of enrichment is called a topological enrichment. By using alternative enrichment scheme, called geometrical enrichment, the size of enriched region can be made independent of element size (Tarancón et al., 2009). The method consists in taking nodes in a specific geometric region, usually a circle of predetermined radius $R$ with its center at a crack tip. Fig. 1 depicts two types of enrichments mentioned above, respectively. In Fig. 1 , nodes marked by a square indicate crack tip enrichment nodes. This Figure also shows the blending elements with dark color around the enriched zone, i.e. elements with only some of their nodes being enriched with singular functions associated with a crack tip asymptotic field. It is generally agreed that choice of enriched nodes could affect significantly on XFEM error. For the topological enrichment, it has been shown that the enrichment by two or three layers give more accurate result as compared with the enrichment by one layer (Liu et al., 2004). In the meantime, the geometrical enrichment can make it to achieve the optimal rate of convergence than the topological one since the smaller the size of element, the more the number of enriched nodes (Tarancón et al., 2009).

\section{Combination of XFEM with LMM for limit analysis}

The general purpose FEA software ANSYS was used to combine XFEM with LMM for limit analysis of cracked structure in this paper. XFEM was implemented by using UserElem in ANSYS for the user-defined element. The 49-point Gauss quadrature was employed for elements containing any 
enriched node while 4-point Gauss quadrature was used for standard elements.

LMM was implemented at the Gauss points in all elements. Thus, LMM needs to implement at 49 Gauss points for enriched nodes and at 4 Gauss points for standard nodes. Young's modulus as well as some quantities for the evaluation of upper and lower were extracted at the Gauss points. Since we do not employ Heaviside enrichment, displacement interpolation for enriched element and standard element according to Eq. (7), respectively, is expressed as follows;

$$
\begin{aligned}
& \mathbf{u}^{h}(\mathbf{x})=\sum_{i \in I} N_{i}(\mathbf{x}) \mathbf{u}_{0 i}+\sum_{k \in M} N_{k}(\mathbf{x})\left\{\sum_{\alpha}\left[F^{\alpha}(\mathbf{x})-F^{\alpha}\left(\mathbf{x}_{k}\right)\right] \mathbf{b}_{k}^{\alpha}\right\}, \\
& \mathbf{u}^{h}(\mathbf{x})=\sum_{i \in I} N_{i}(\mathbf{x}) \mathbf{u}_{0_{i}} .
\end{aligned}
$$

Numerical accuracy of limit analysis can be improved as compared with standard FEM since mechanical quantities near a crack tip, which plays a dominant role for the evaluation of upper and lower of cracked structure can be evaluated more precisely by enrichment. Eq. (10) could be reduced into standard FEM if an enriched degree of freedom $\mathbf{b}_{k}^{\alpha}$ does not exist or be equal to zero. Therefore, limit analysis by standard FEM can be considered as a special case of limit analysis by XFEM where an enriched degree of freedom $\mathbf{b}_{k}^{\alpha}$ is restricted by zero. LMM was implemented by using UserMat in ANSYS for user-defined material. Since UserElem calls UserMat automatically, one does not need to pay an attention for mutual exchange between the two. LMM for standard elements was also implemented by using user-defined element. FE modeling can be done easily as standard functions of ANSYS could be employed for FE mesh of user-defined elements, too.

\section{Numerical examples}

In this study, we applied FE mesh matched with the crack geometry where crack faces are coincident with element edges and a crack tip lies on a node in order to exclude the effect of discontinuous enrichment of displacement. The topological enrichment with two layers is used for the comparison of standard FEM with XFEM while the geometrical enrichment is employed for considering the effect of size of enriched zone. The size of geometrically enriched zone is set as onetwentieth of crack length.

\subsection{Single-edge tension specimen $(S E(T))$}

Fig. 2 shows a geometry of SE(T) specimen. Plane strain state is assumed. Geometric parameters with $b=1$ and $L=b$ are applied. For plane stress state, analytical solution for limit load factor $\lambda=p / \sigma_{y}$ is expressed as

$$
\begin{aligned}
& \lambda=\left[\left(1-\gamma x+\frac{\gamma-1}{2}\right)^{2}+\gamma(1-x)^{2}\right]^{1 / 2}-\left(\gamma x-\frac{\gamma-1}{2}\right), x>0.146, \\
& \lambda=1-x-x^{2}, x \leq 0.146
\end{aligned}
$$

where, $x=a / b$ and $\gamma$ is equal to $2 / \sqrt{3}$ for von Mises yielding criterion and 1 for Tresca yielding criterion (Ewing \& Richards, 1974). 

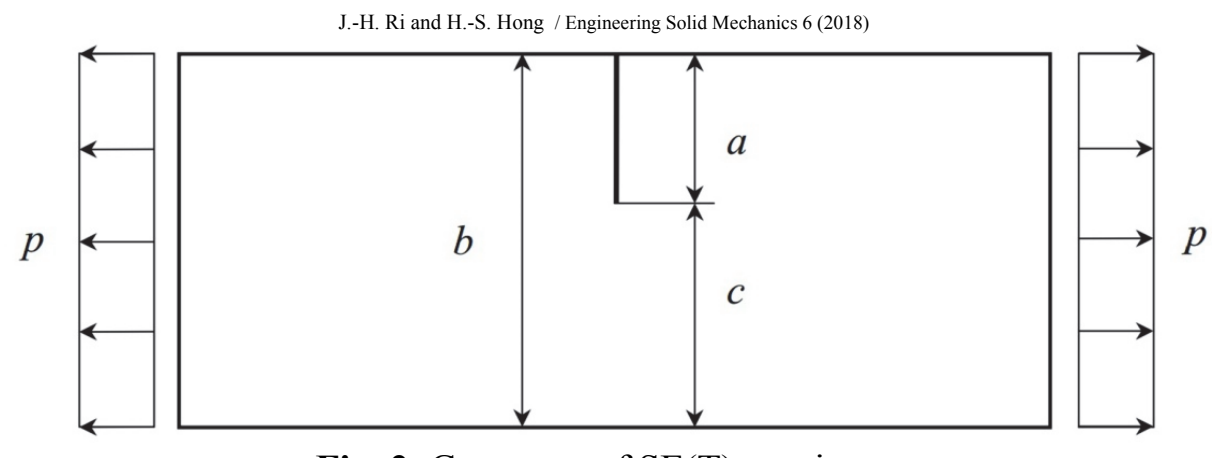

Fig. 2. Geometry of SE(T) specimen

For comparison of numerical accuracy, iteration number is fixed by 30 . First let us consider the effect of element size for $\mathrm{SE}(\mathrm{T})$ specimen with $a / b=0.6$. Due to symmetry, only the half FE model is used and FE mesh adopts square elements. Fig. 3 shows the FE mesh with element size of $b / 50$.

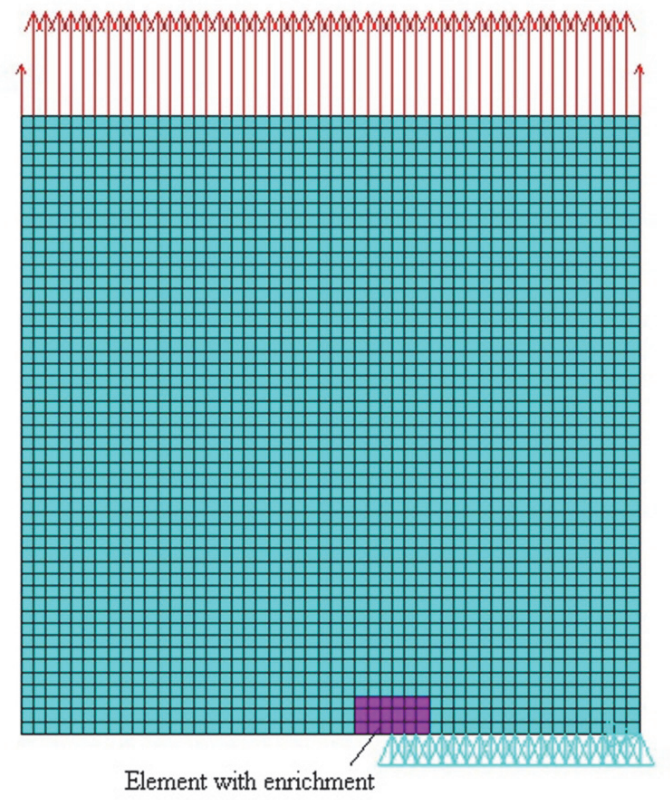

Fig. 3. FE mesh for $\mathrm{SE}(\mathrm{T})$ specimen with $a / b=0.6$

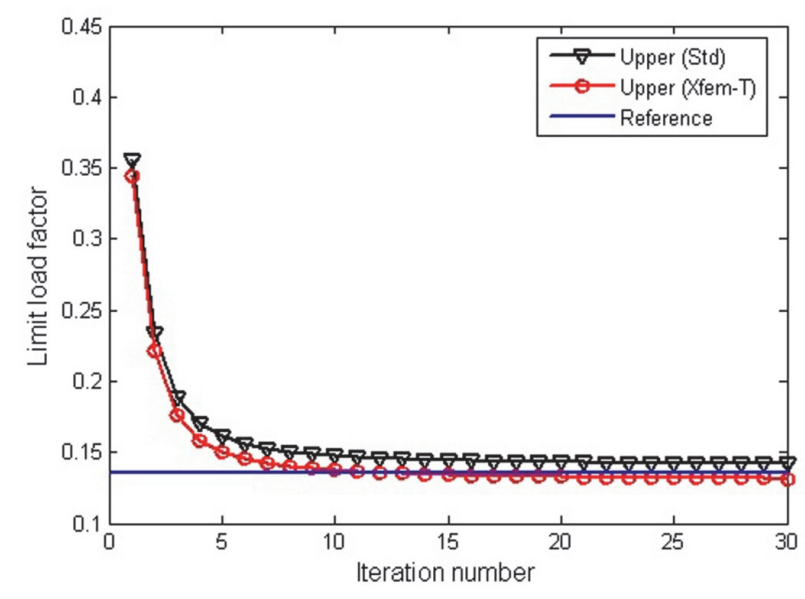

Fig. 4. Convergence process of a factor of limit load for $\operatorname{SE}(\mathrm{T})$ specimen with $a / b=0.6$

Analytical factor of limit load is equal to $\lambda=0.1352$ according to Eq. (12). Fig. 4 shows convergence process of a factor of limit load with iteration number calculated by XFEM with topological enrichment of two layers and standard FEM for element size of $b / 50$, respectively. In this figure, curves calculated using standard FEM are marked by a symbol "std" and symbol "Xfem-T" means curves calculated using XFEM with topological enrichment of 2 layers. As shown from this figure, LMM combined with XFEM gives more accurate result than the one combined with standard FEM although both have good convergence. Moreover, XFEM gives lower values of limit load factor than analytical solution whereas standard FEM predicts higher values than analytical one, thus XFEM will be preferable from the viewpoint of structural safety.

Fig. 5 shows the effect of element size on a factor of limit load in $\operatorname{SE}(\mathrm{T})$ specimen with $a / b=0.6$ for LMM combined with XFEM and with standard FEM. 

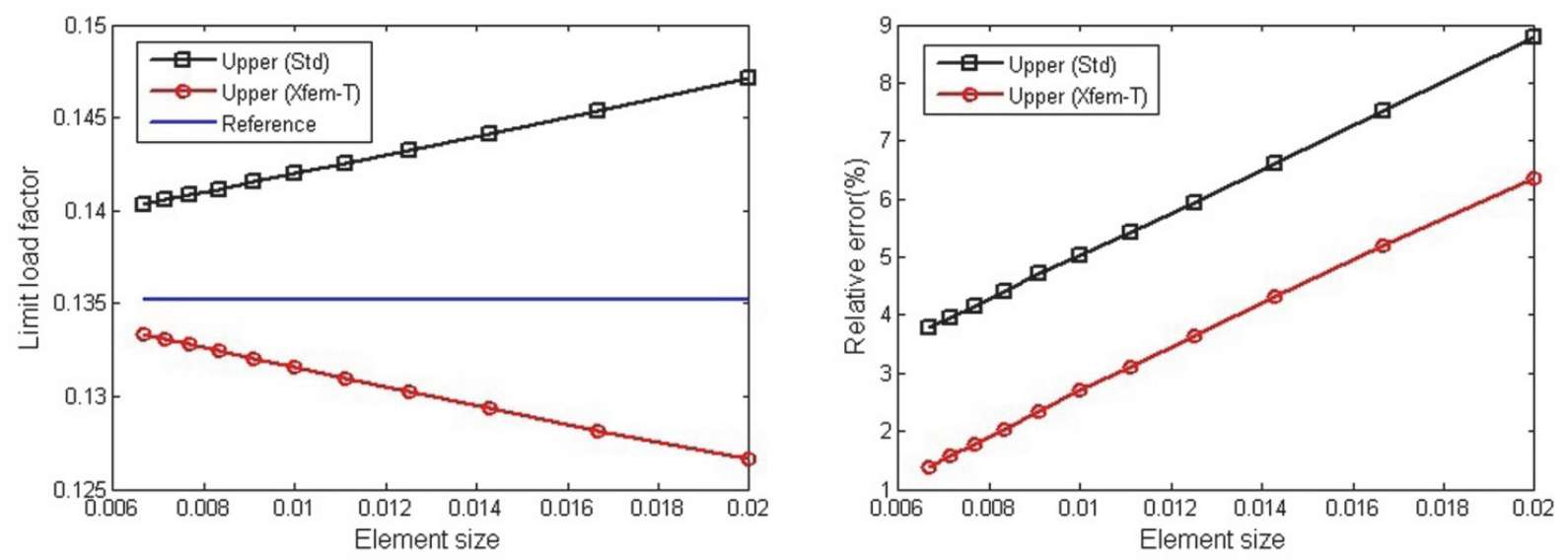

Fig. 5. Effect of element size on a factor of limit load for SE(T) specimen with $a / b=0.6$

As seen from this figure, a factor of limit load calculated by standard FEM as well as XFEM significantly depends on the element size. XFEM with topological enrichment of two layers gives lower values of a factor of limit load than analytical solution while standard FEM predicts higher ones than analytical solution, but XFEM shows higher numerical accuracy with 2 or 3 percentage as well as more rapid convergence rate as compared with standard FEM. In order to see the effect of enrichment type on the numerical accuracy of XFEM combined with LMM, geometrical enrichment is applied, setting enriched zone as a circle with a radius of $R$. Fig. 6 compares a limit load factor for topological enrichment of two layers with the one for geometrical enrichment with a circle of a radius of $R=a / 20$, varying the element size for $\mathrm{SE}(\mathrm{T})$ specimen with a relative crack size of $a / b=0.6$. Here, Xfem-G depicts results for geometrical enrichment. As one can see from Fig. 6, geometrical enrichment does not show the remarkable tendency as compared with topological enrichment, but both have the similar effect of element size on the numerical accuracy.
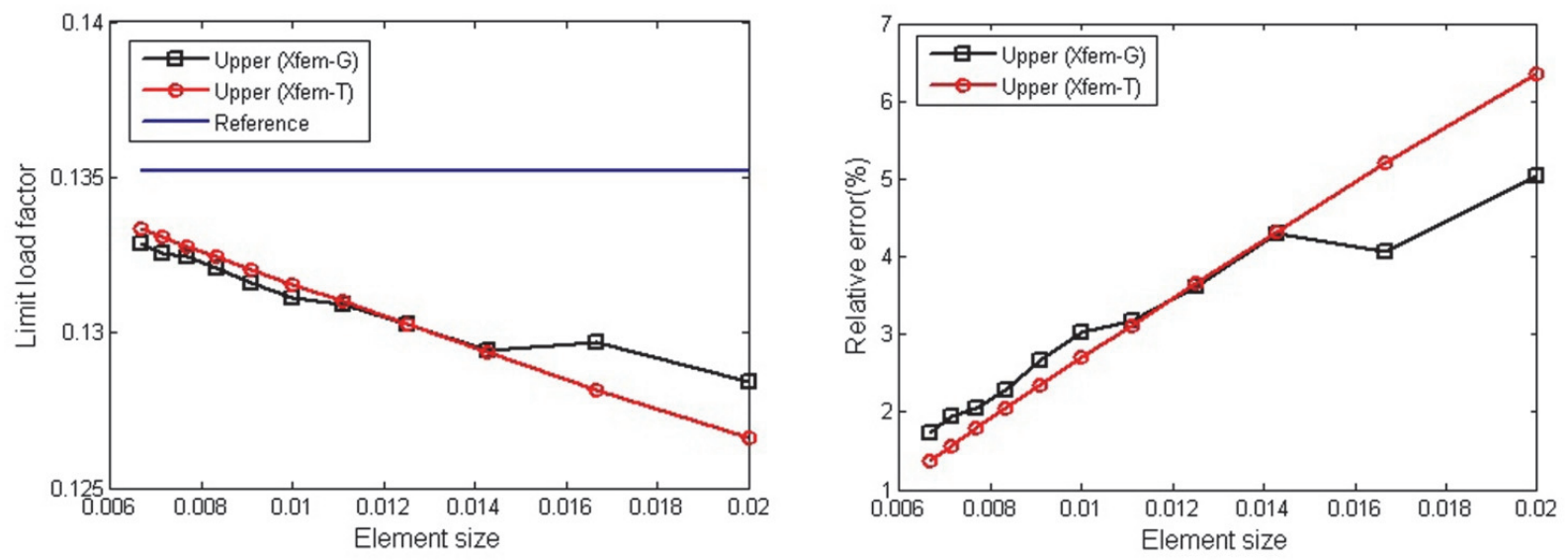

Fig. 6. Effect of enrichment type on a factor of limit load for SE(T) specimen with $a / b=0.6$

Fig. 7 shows the error of limit load factor versus the size of enriched zone $R / a$ for with a relative crack size of $a / b=0.3$ and 0.6 , respectively. As seen from this figure, the smaller the size of enriched zone, the higher the numerical accuracy of limit load for $a / b=0.6$. But the situation is not the same for $a / b=0.3$. This means that XFEM requires different selections of reasonable enriched zone, depending on the characteristics of specified problem. Nevertheless, XFEM gave more accurate result than standard FEM, irrespective of the size of enriched zone. In order to see the effect of a crack depth on the numerical accuracy of limit load factor, FE mesh with the element size of $b / 100$ was applied for different crack depths. Fig. 8 shows the variation of a load limit factor and its relative error as crack depth is increased. As shown from this figure, XFEM combined with LMM estimates a limit load factor 
more correctly for different crack depths than standard FEM.
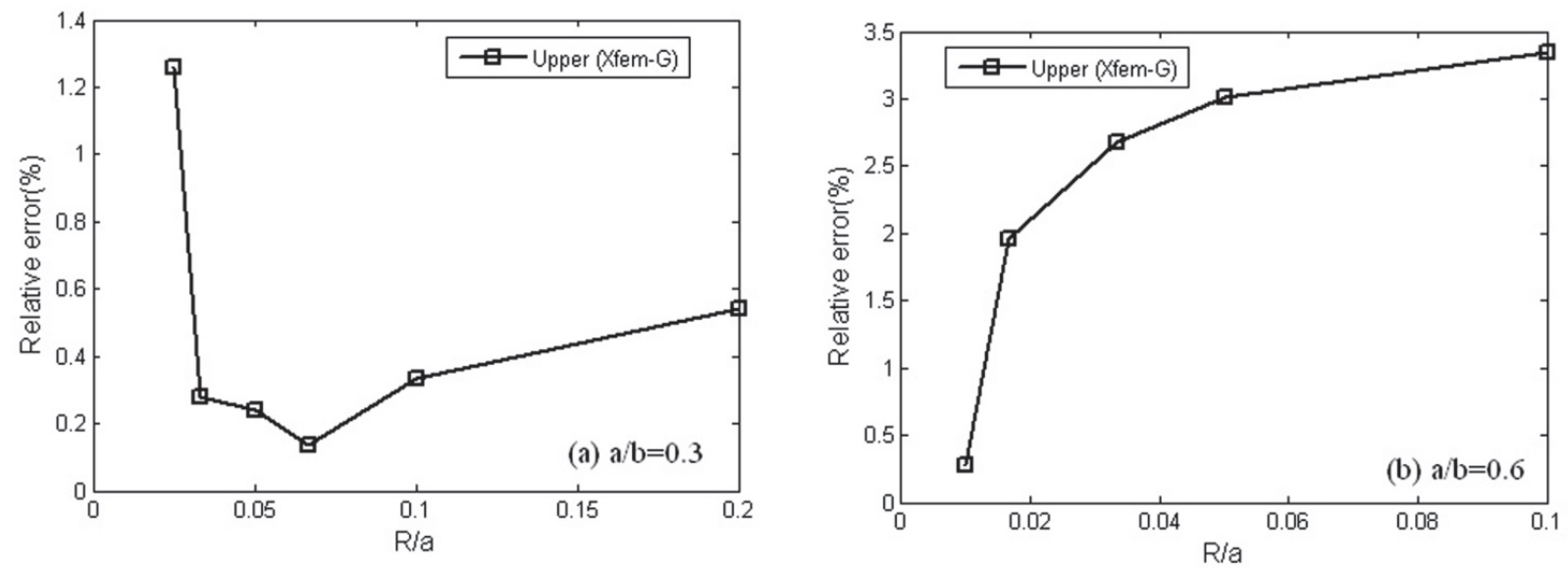

Fig. 7. Effect of size $(R / a)$ of enriched zone on a limit load factor: (a) $a / b=0.3$; (b) $a / b=0.6$
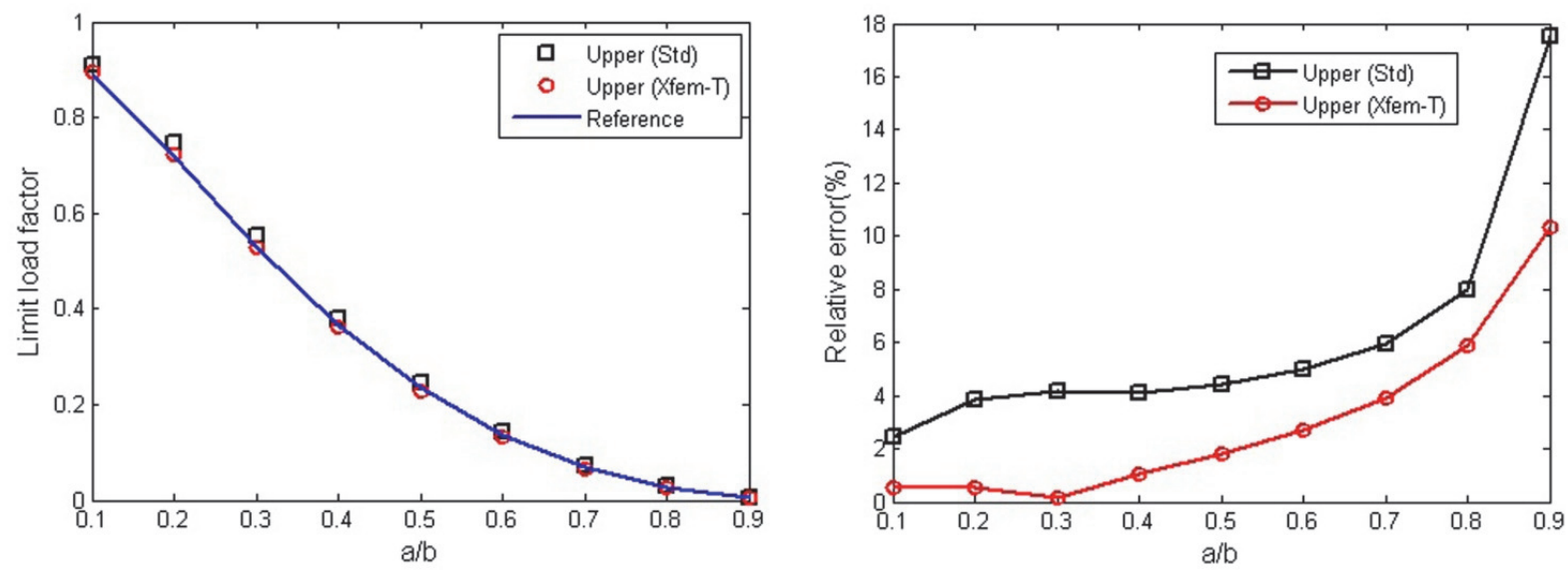

Fig. 8. Variation of a load limit factor and its relative error for different crack depths

\subsection{Center-cracked tension specimen (CCT)}

Fig. 9 shows the geometry of CCT specimen. Plane stress state is assumed while geometric parameters with $b=1$ and $L=b$ are applied. Analytical solution of a load limit factor $\lambda=p / \sigma_{y}$ for CCT specimen in case of plane stress state is expressed as follows (Miller, 1988).

$$
\lambda=1-\frac{a}{b}
$$

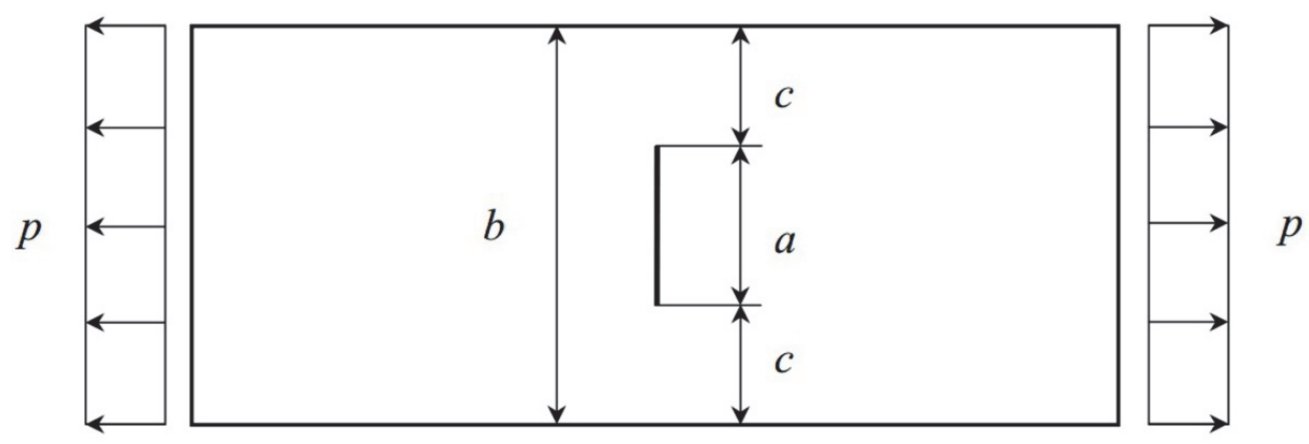

Fig. 9. Geometry of CCT specimen. 
$\mathrm{SE}(\mathrm{T})$ specimen with $a / b=0.5$ is considered in order to see the effect of element size on the numerical accuracy of a load limit factor. Due to symmetry, only the quarter FE model is used and FE mesh adopts square elements. As in the SE(T) specimen, a limit load factor is calculated by 30 iterations. Fig. 10 shows FE mesh with the element size of $b / 50$.

The analytical limit load factor is equal to $\lambda=0.5$ according to Equation (13). XFEM evaluates a limit load factor as 0.4977 while standard FEM as 0.5214 , leading to the relative error of 0.46 and 4.28 percents, respectively. Figure 11 shows the convergence process of a limit load factor calculated by standard FEM and XFEM with topological enrichment of two layers for the element size of $b / 50$ as the iteration number is increased, respectively. As shown above, LMM combined with XFEM gives more accurate result than one combined with standard FEM like SE(T) specimen.

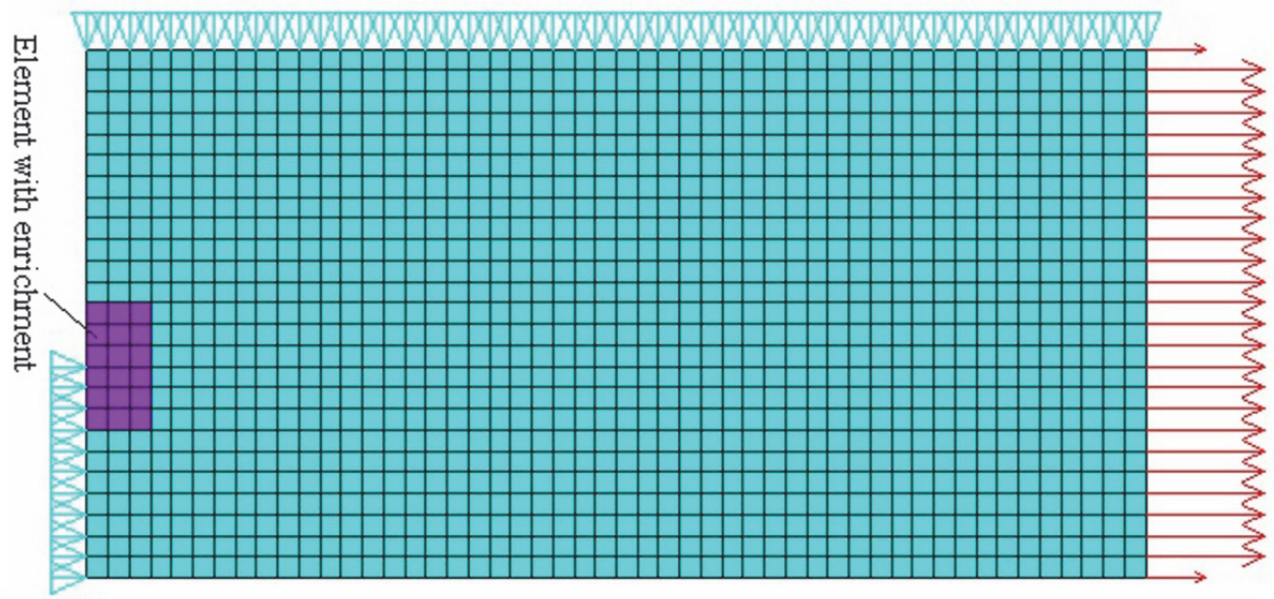

Fig. 10. FE mesh for CCT specimen with $a / b=0.5$

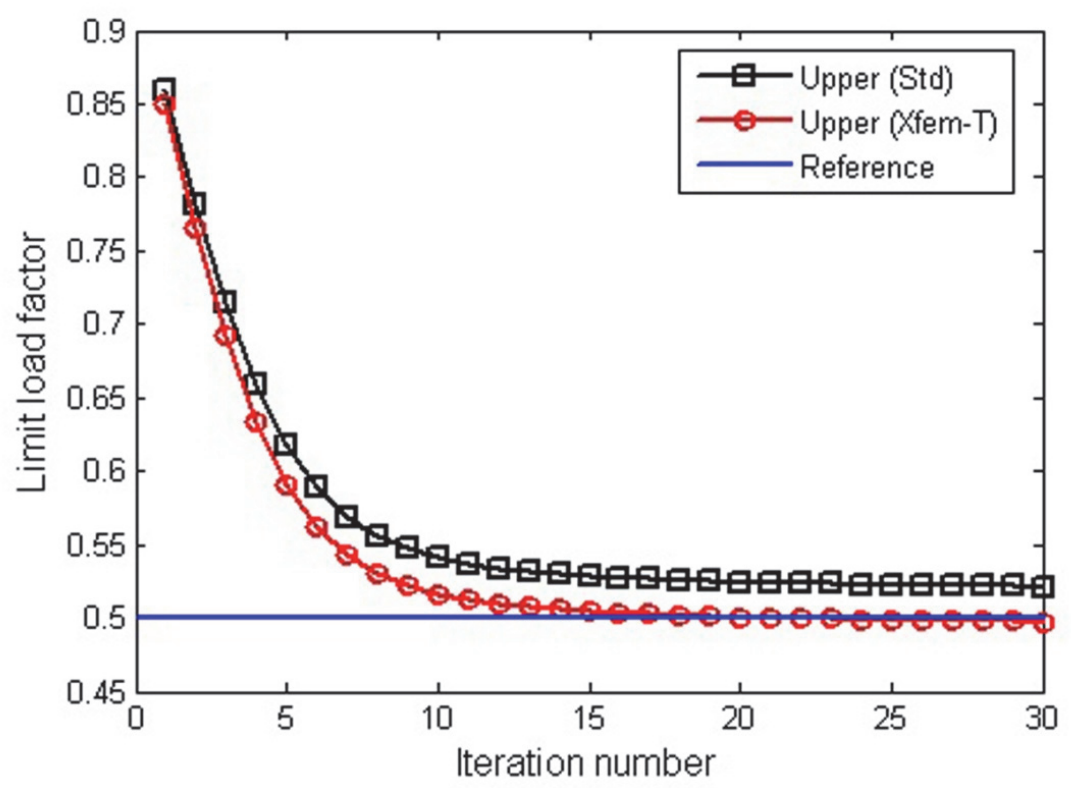

Fig. 11. Convergence process of a limit load factor for CCT specimen with $a / b=0.5$

Fig. 12 shows the effect of element size on the upper bound of a factor of limit load in CCT specimen for LMM combined with XFEM and with standard FEM. From this figure, one can easily see the same tendency for the effect of element size as in SE(T) specimen. In the meantime, XFEM shows the higher numerical accuracy with 3 5\% than standard FEM as well as the more rapid convergence rate as compared with standard FEM. 

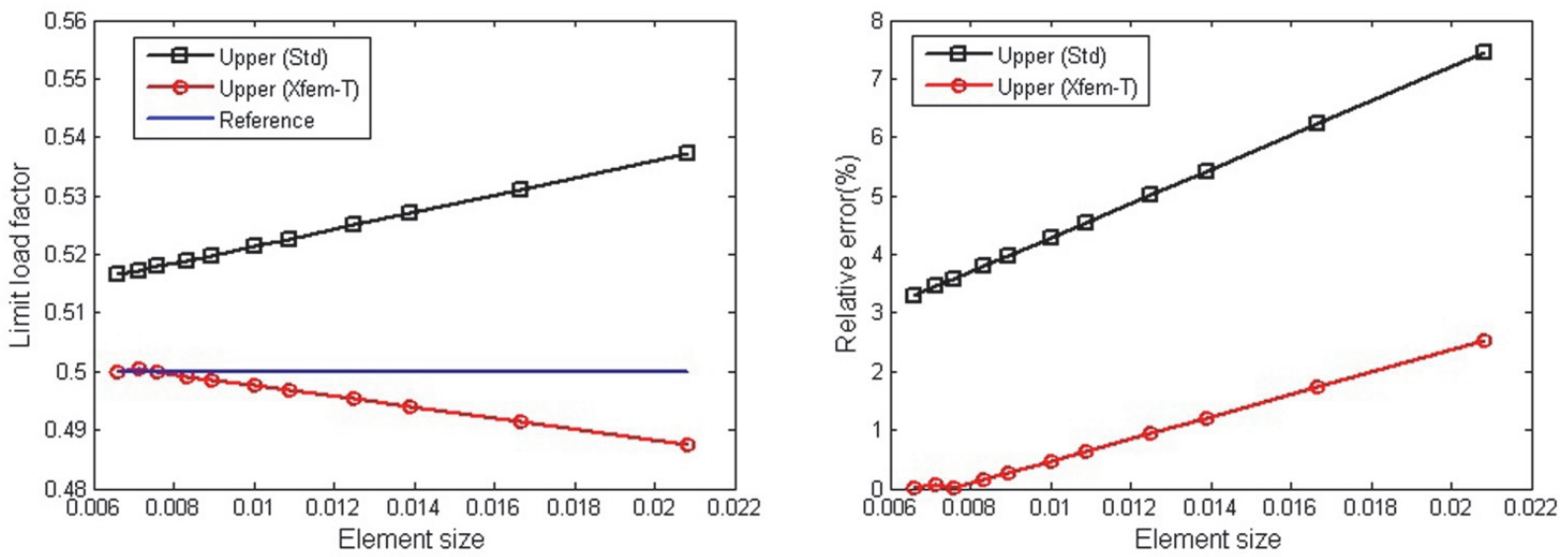

Fig. 12. Effect of element size on a factor of limit load for CCT specimen with $a / b=0.5$

In order to consider the effect of size of enriched zone on the numerical accuracy of XFEM combined with LMM, geometrical enrichment is applied, setting the enriched zone as a circle with a radius of $R$. Fig. 13 compares a limit load factor for topological enrichment of two layers with one for geometrical enrichment with a circle of a radius of $R=a / 20$, varying the element size for CCT specimen.
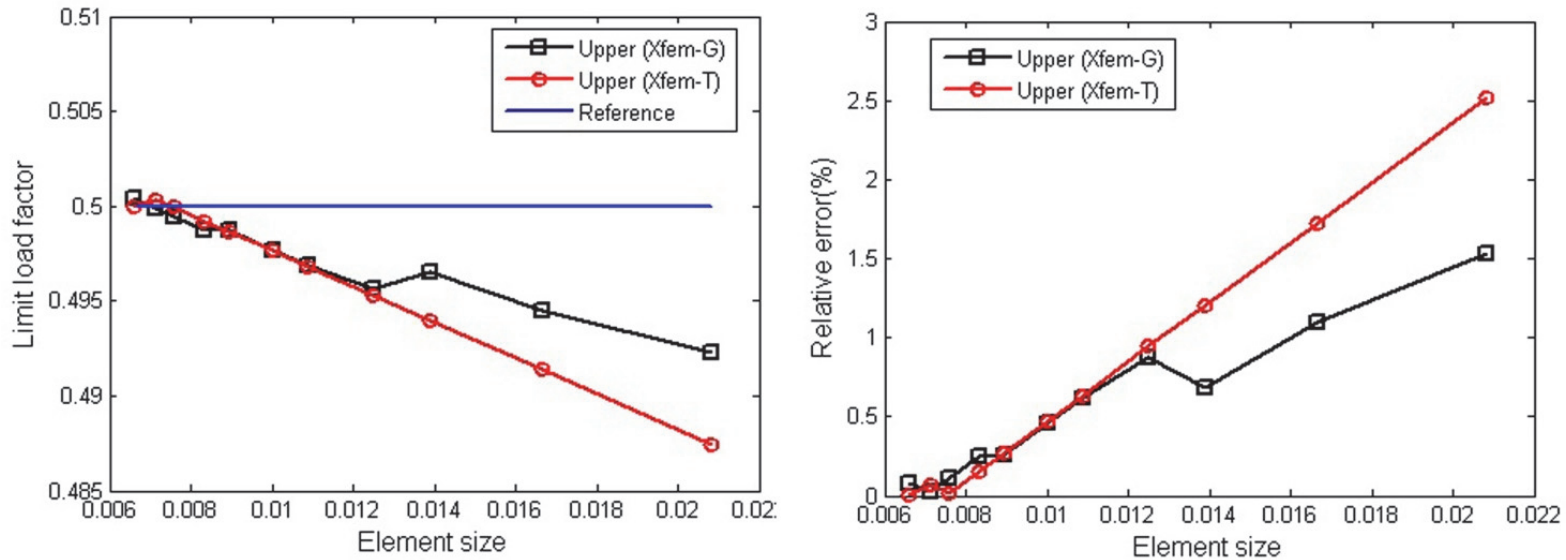

Fig. 13. Effect of enrichment type on a factor of limit load for CCT specimen with $a / b=0.5$
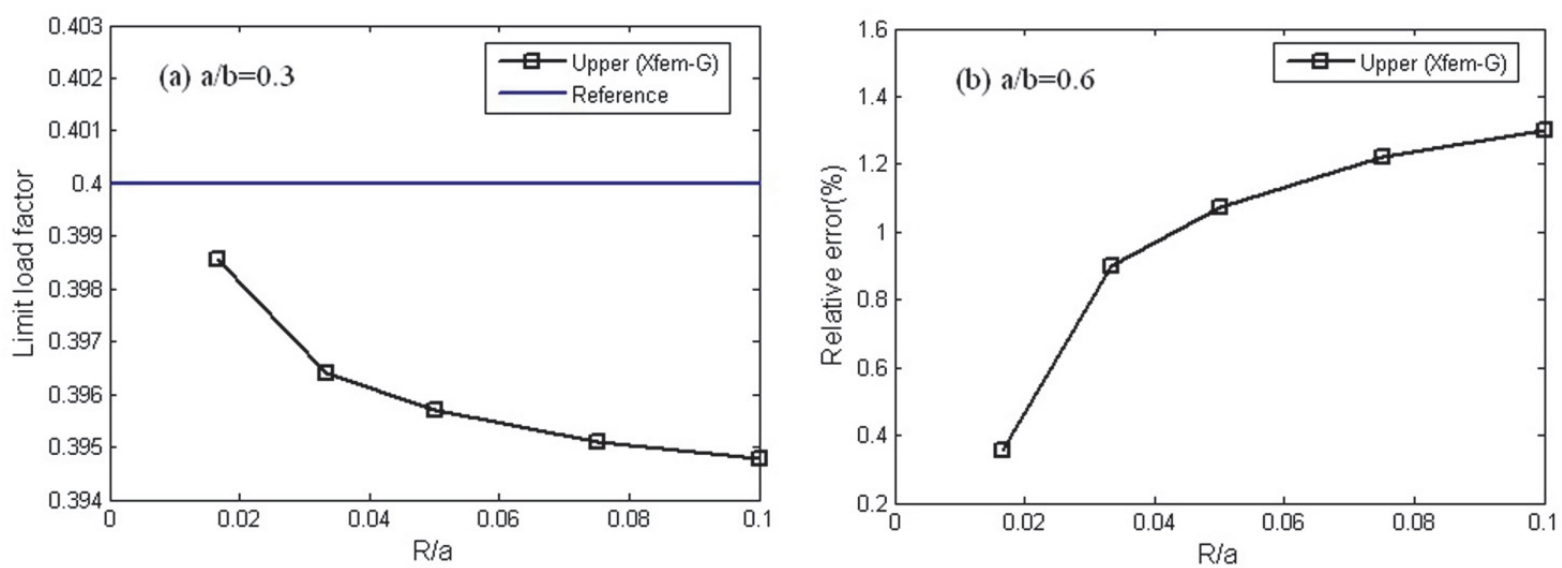

Fig. 14. Effect of size $R$ of enriched zone on a limit load factor: (a) $a / b=0.3$; (b) $a / b=0.6$

As one can see from Fig. 13, geometrical enrichment has the similar tendency for the effect of element size on a limit load factor as in topological enrichment. Fig. 14 shows the relative error of 
upper bound of a limit load factor versus normalized size of enriched zone $R / a$ with the element size of $b / 100$ for relative crack length $a / b$ of 0.3 and 0.6 , respectively. As seen from this figure, size of enriched zone can affect significantly the numerical accuracy of a limit load factor and its tendency depends on crack length. Likewise $\mathrm{SE}(\mathrm{T})$ specimen, the smaller the enriched zone the higher the numerical accuracy of a limit load factor for case of $a / b=0.6$ while the relative error has the inflection point for case of $a / b=0.3$. This means that the small size of enriched zone is preferable for deep crack whereas the relative error becomes greater if the size of enriched zone is smaller than a certain value for shallow crack.
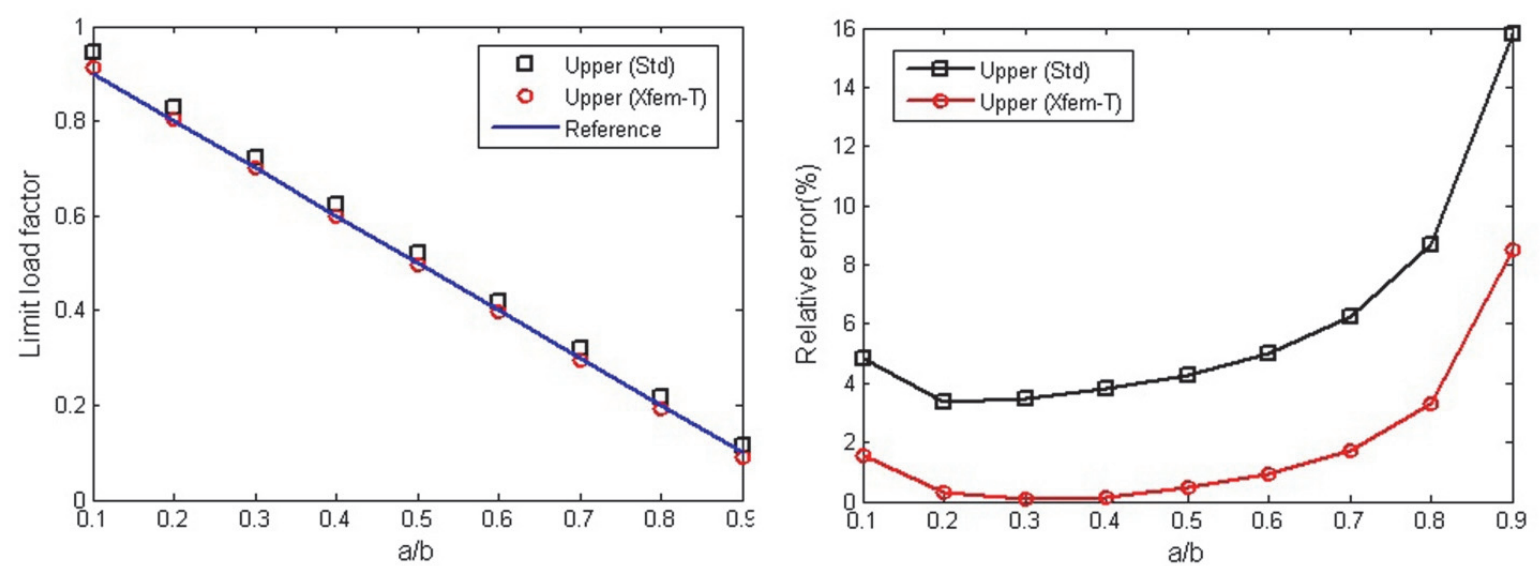

Fig. 15. Variation of a load limit factor and its relative error with different crack depths.

In order to see the effect of crack depth on the numerical accuracy of a limit load factor, FE mesh with the element size of $b / 100$ was applied for different crack depths. Fig. 15 shows the variation of upper bound of load limit factor and its relative error as the crack depth is increased. As shown from this figure, XFEM combined with LMM improves the numerical accuracy of a limit load factor as compared with standard FEM combined with LMM

\section{Discussion and conclusion}

In this paper, we studied the limit analysis of cracked structure by the combination of XFEM with LMM. Numerical results for SE(T) and CCT specimens demonstrated that the combination of XFEM with LMM is an appropriate method for the limit analysis of cracked structure. XFEM combined with LMM improved the numerical accuracy of limit analysis than standard FEM combined with one. XFEM always gave the more accurate limit load factor as well as the more rapid convergence rate in all cases considered in this paper. Furthermore, it should be noted that the size of enriched zone plays an important role for the improvement of numerical accuracy for the limit analysis of cracked structure. In general, the reasonable size of enriched zone depends on the details of specified problem. The small size of enriched zone is preferable for deep crack whereas the relative error becomes greater if the size of enriched zone is smaller than a certain value for shallow crack. Nevertheless, XFEM gave the more accurate result than standard FEM, independently of the size of enriched zone. Thus, one can conclude that XFEM improves the limit analysis of cracked structure significantly than the standard FEM. Only, its effectiveness depends on the size of enriched zone. It should be also noted that the shakedown analysis of cracked structure by XFEM combined with LMM needs future study.

\section{Acknowledgement}

The authors would like to express their deep gratitude to Professor A.R.S Ponter of University of Leicester, UK for giving us valuable comments on this work. Also, the authors gratefully acknowledge Professor D. Weichert of RWTH Aachen University, German for encouraging this work and giving some advices. 


\section{References}

Barrera, O., Cocks, A. C. F., \& Ponter, A. R. S. (2011). The linear matching method applied to composite materials: a micromechanical approach. Composites Science and Technology, 71(6), 797804.

Belytschko, T., \& Black, T. (1999). Elastic crack growth in finite elements with minimal remeshing. International journal for numerical methods in engineering, 45(5), 601-620.

Casciaro, R., \& Garcea, G. (2002). An iterative method for shakedown analysis. Computer methods in applied mechanics and engineering, 191(49), 5761-5792.

Chen, H., Chen, W., Li, T., \& Ure, J. (2011). Effect of circular holes on the ratchet limit and crack tip plastic strain range in a centre cracked plate. Engineering Fracture Mechanics, 78(11), 2310-2324.

Chen, H., \& Ponter, A. R. (2001). A method for the evaluation of a ratchet limit and the amplitude of plastic strain for bodies subjected to cyclic loading. European Journal of Mechanics-A/Solids, 20(4), 555-571.

Chen, H. F., \& Ponter, A. R. (2005a). Integrity assessment of a 3D tubeplate using the linear matching method. Part 1. Shakedown, reverse plasticity and ratchetting. International Journal of Pressure Vessels and Piping, 82(2), 85-94.

Chen, H. F., \& Ponter, A. R. (2005b). Integrity assessment of a 3D tubeplate using the linear matching method. Part 2: Creep relaxation and reverse plasticity. International journal of pressure vessels and piping, 82(2), 95-104.

Chen, H. F., \& Ponter, A. R. (2005c). The linear matching method for shakedown and limit analyses applied to rolling and sliding point contact problems. Road materials and pavement design, 6(1), 930.

Chen, H., \& Ponter, A. R. (2006). Linear Matching Method on the evaluation of plastic and creep behaviours for bodies subjected to cyclic thermal and mechanical loading. International Journal for Numerical Methods in Engineering, 68(1), 13-32.

Chen, H., \& Ponter, A. R. (2009). Structural integrity assessment of superheater outlet penetration tubeplate. International Journal of Pressure Vessels and Piping, 86(7), 412-419.

Chen, S., Liu, Y., \& Cen, Z. (2008). Lower-bound limit analysis by using the EFG method and nonlinear programming. International Journal for Numerical Methods in Engineering, 74(3), 391-415.

Ciria, H., Peraire, J., \& Bonet, J. (2008). Mesh adaptive computation of upper and lower bounds in limit analysis. International journal for numerical methods in engineering, 75(8), 899-944.

Ewing, D. J. F., \& Richards, C. E. (1974). The yield-point loads of singly-notched pin-loaded tensile strips. Journal of the Mechanics and Physics of Solids, 22(1), 27-36.

Fries, T. P., \& Belytschko, T. (2010). The extended/generalized finite element method: an overview of the method and its applications. International Journal for Numerical Methods in Engineering, 84(3), 253-304.

Fuschi, P., Pisano, A. A., \& Barrera, O. (2009). Limit analysis of orthotropic laminates by Linear Matching Method. Limit States of Materials and Structures-Direct Methods, 197-220.

Gorash, Y., \& Chen, H. (2013). A parametric study on creep-fatigue strength of welded joints using the linear matching method. International Journal of Fatigue, 55, 112-125.

Moës, N., Gravouil, A., \& Belytschko, T. (2002). Non-planar 3D crack growth by the extended finite element and level sets-Part I: Mechanical model. International Journal for Numerical Methods in Engineering, 53(11), 2549-2568.

Habibullah, M. S., \& Ponter, A. R. S. (2005). Ratchetting limits for cracked bodies subjected to cyclic loads and temperatures. Engineering fracture mechanics, 72(11), 1702-1716.

Huang, R., Sukumar, N., \& Prévost, J. H. (2003). Modeling quasi-static crack growth with the extended finite element method Part II: Numerical applications. International Journal of Solids and Structures, 40(26), 7539-7552.

Le, C. V., Gilbert, M., \& Askes, H. (2009). Limit analysis of plates using the EFG method and secondorder cone programming. International Journal for Numerical Methods in Engineering, 78(13), $1532-1552$. 
Le, C. V., Gilbert, M., \& Askes, H. (2010). Limit analysis of plates and slabs using a meshless equilibrium formulation. International Journal for Numerical Methods in Engineering, 83(13), 1739-1758.

Liu, X. Y., Xiao, Q. Z., \& Karihaloo, B. L. (2004). XFEM for direct evaluation of mixed mode SIFs in homogeneous and bi-materials. International Journal for Numerical Methods in Engineering, 59(8), 1103-1118.

Miller, A. G. (1988). Review of limit loads of structures containing defects. International Journal of Pressure Vessels and Piping, 32(1), 197-327.

Dolbow, J. O. H. N., \& Belytschko, T. (1999). A finite element method for crack growth without remeshing. International journal for numerical methods in engineering, 46(1), 131-150.

Moës, N., Gravouil, A., \& Belytschko, T. (2002). Non-planar 3D crack growth by the extended finite element and level sets-Part I: Mechanical model. International Journal for Numerical Methods in Engineering, 53(11), 2549-2568.

Munoz, J. J., Bonet, J., Huerta, A., \& Peraire, J. (2009). Upper and lower bounds in limit analysis: adaptive meshing strategies and discontinuous loading. International Journal for Numerical Methods in Engineering, 77(4), 471-501.

Ponter, A. R., Chen, H., Willis, M. R., \& Evans, W. J. (2004). Fatigue-creep and plastic collapse of notched bars. Fatigue \& Fracture of Engineering Materials \& Structures, 27(4), 305-318.

Staat, M., \& Heitzer, M. (2003). Numerical methods for limit and shakedown analysis. Series of John von Neumann institute for computing, 15, 306.

Sukumar, N., \& Prévost, J. H. (2003). Modeling quasi-static crack growth with the extended finite element method Part I: Computer implementation. International journal of solids and structures, 40(26), 7513-7537.

Tarancón, J. E., Vercher, A., Giner, E., \& Fuenmayor, F. J. (2009). Enhanced blending elements for XFEM applied to linear elastic fracture mechanics. International Journal for Numerical Methods in Engineering, 77(1), 126-148.

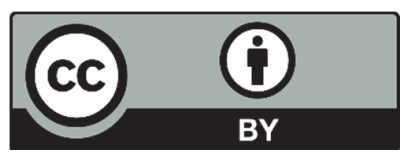

(C) 2018 by the authors; licensee Growing Science, Canada. This is an open access article distributed under the terms and conditions of the Creative Commons Attribution (CC-BY)

license (http://creativecommons.org/licenses/by/4.0/). 